Supporting Information

\title{
Post-Cross-linking of Collagen Hydrogels by Carboxymethylated Polyrotaxanes for Simultaneously Improving Mechanical Strength and Cell Proliferation
}

Atsushi Tamura $^{1, *}$, Dae Hoon Lee ${ }^{1,2}$, Yoshinori Arisaka ${ }^{1}$, Tae Woong Kang ${ }^{1}$, Nobuhiko Yui ${ }^{1}$

${ }^{1}$ Department of Organic Biomaterials, Institute of Biomaterials and Bioengineering, Tokyo Medical and Dental University (TMDU), 2-3-10 Kanda-Surugadai, Chiyoda, Tokyo 101-0062, Japan.

${ }^{2}$ Department of Bionanotechnology and Bio-Convergence Engineering, Jeonbuk National University, 567 Baekje-daero, Deokjin-gu, Jeonju, Jeonbuk 54896, Republic of Korea.

*Corresponding author: Atsushi Tamura (E-mail: tamura.org@tmd.ac.jp) 


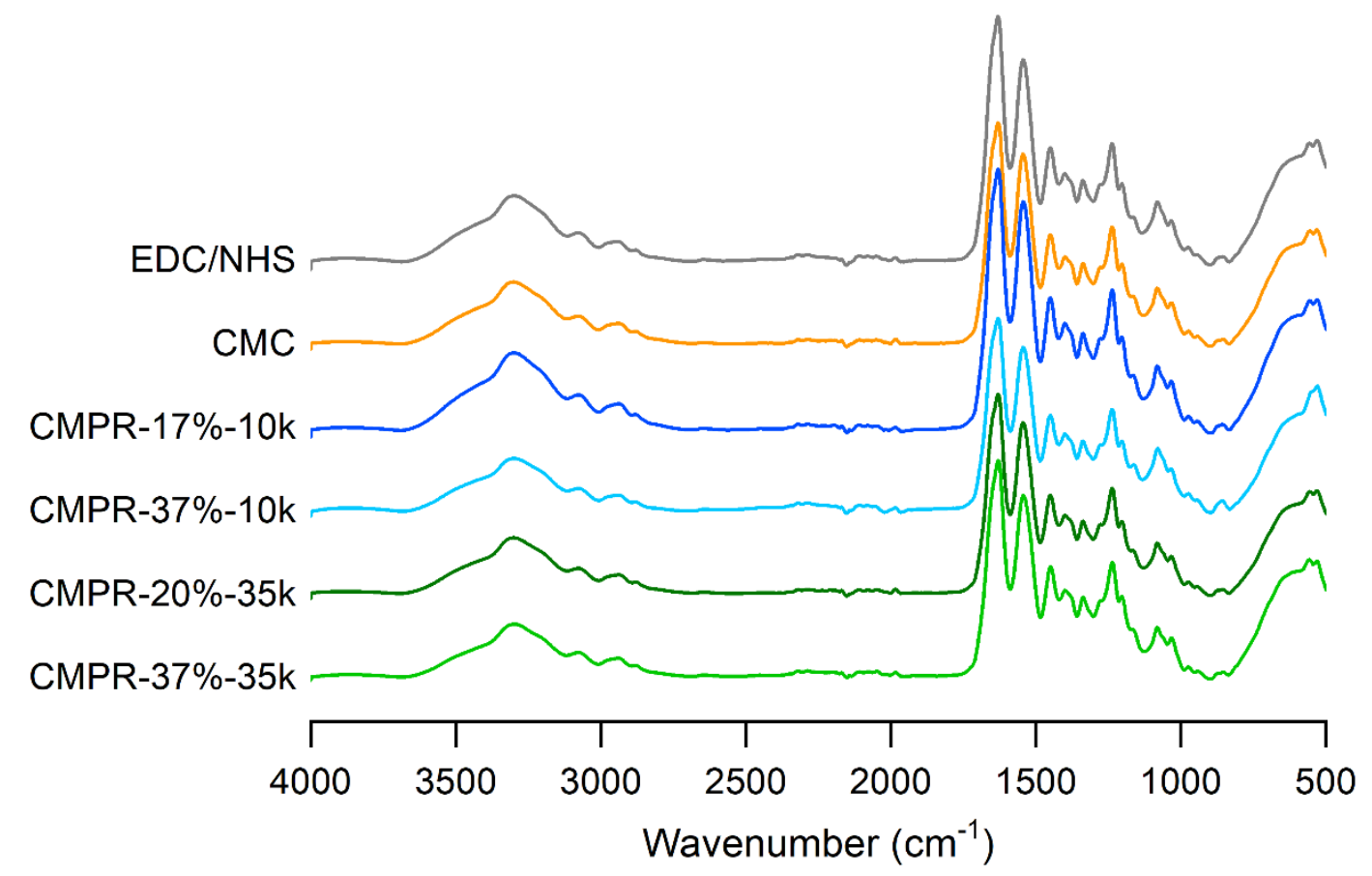

Figure S1. ATR-FTIR spectra of collagen hydrogels post-cross-linked with EDC/NHS, CMC, and CMPRs.

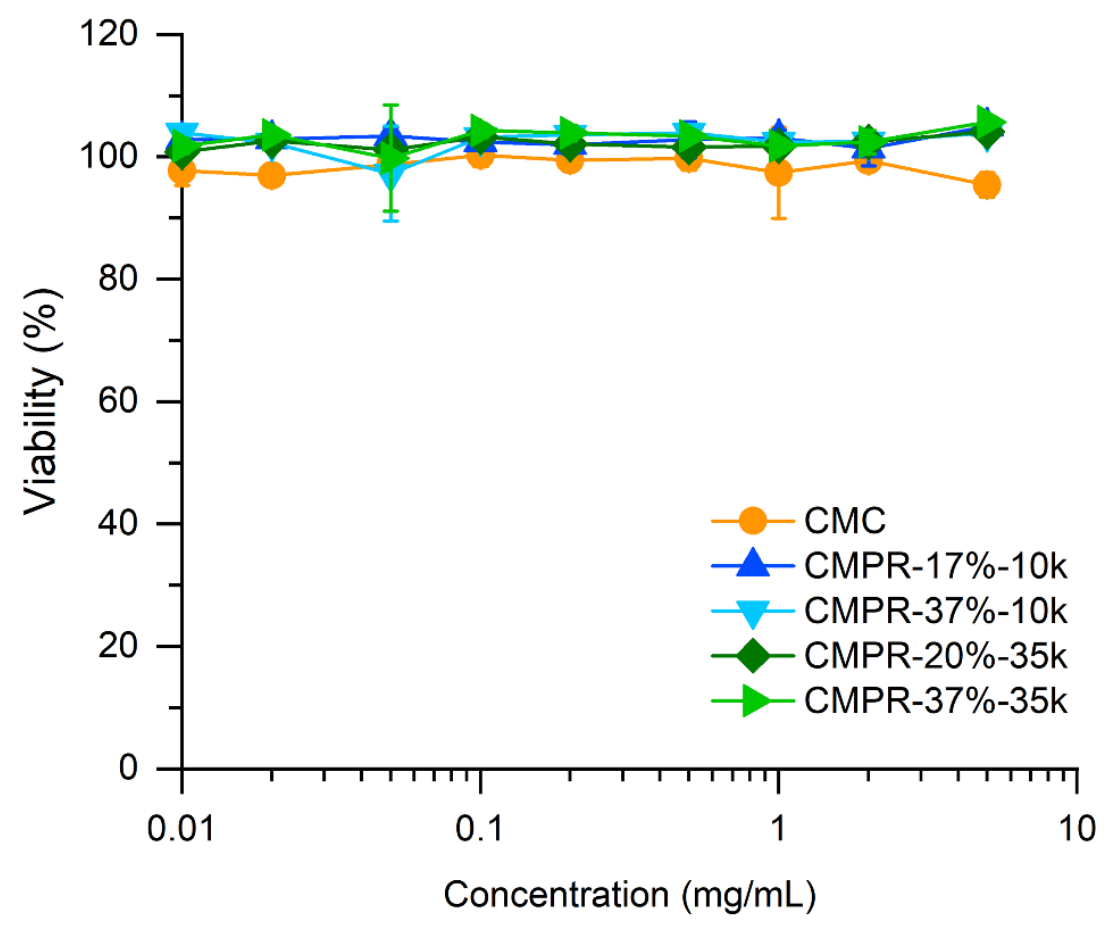

Figure S2. Viability of BALB/3T3 cells treated with CMC and CMPRs for $24 \mathrm{~h}$. Data are expressed as mean $\pm \operatorname{SD}(n=5)$. 